\title{
Modeling of thermal sensitivity of a fiber optic gyroscope coil with practical quadrupole winding
}

\section{Serdar Ogut, Berk Osunluk, Ekmel Ozbay}

Serdar Ogut, Berk Osunluk, Ekmel Ozbay, "Modeling of thermal sensitivity of a fiber optic gyroscope coil with practical quadrupole winding," Proc. SPIE 10208, Fiber Optic Sensors and Applications XIV, 1020806 (27 April 2017); doi: $10.1117 / 12.2258030$

SPIE Event: SPIE Commercial + Scientific Sensing and Imaging, 2017, Anaheim, California, United States 


\title{
Modeling of thermal sensitivity of a fiber optic gyroscope coil with practical quadrupole winding
}

\author{
Serdar Ogut*a,b, Berk Osunluk ${ }^{\mathrm{a}, \mathrm{c}}$, and Ekmel Ozbay ${ }^{\mathrm{a}, \mathrm{b}}$ \\ ${ }^{a}$ Dept. of Electrical and Electronics Engineering, Bilkent University, 06800, Ankara, Turkey \\ ${ }^{\mathrm{b} N a n o t e c h n o l o g y}$ Research Center - NANOTAM, Bilkent University, 06800, Ankara, Turkey \\ ${ }^{c}$ Aselsan Inc., Microelectronics, Guidance and Electro-optics Sector, 06011, Ankara, Turkey
}

\begin{abstract}
Thermally induced bias error is one of the main performance limits for the fiber optic gyroscopes (FOGs). We reviewed the thermal sensitivity of FOG in detail and created a simulation environment by the Finite Element Method (FEM). Thermal sensitivity analysis is based on Shupe and elastooptic effects. Elastooptical interactions are modeled by using the two different FEM simulations and homogenization-dehomogenization processes. FEM simulations are validated by comparing the results with a laboratory FOG setup. We report the changes in the error characteristics for practical quadruple winding patterns.
\end{abstract}

Keywords: Fiber optic gyroscope (FOG), fiber sensing coil, Shupe effect, elastooptical interactions, finite element method (FEM), quadrupole winding, homogenization, dehomogenization

\section{INTRODUCTION}

Thermal effects have been the major precision limit for fiber optic gyroscope (FOG) technology. Thermally induced nonreciprocal phase shift in fiber optic interferometer is first introduced by Shupe. ${ }^{1}$ It is followed by the invention of symmetrical winding methods to reduce this effect. ${ }^{2}$ One of most widely used symmetrical winding is the quadrupole pattern. Another major nonreciprocal phase shift mechanism, elastooptical phase shift, which is indirectly induced by thermal fluctuations is also proposed in Ref. 3. Thermal fluctuations create stress and strain on the fiber core resulting a phase shift. The stress field on fiber is effected by fiber structure, adhesive between fibers, spool material and structure. Effect of each component is not independent so it is complicated to calculate the resulting phase shift. We build a model including all thermal and elastooptical interactions, and calculated the induced bias error with the assumption of nonideal practical quadrupole winding. We compared the calculated bias errors with three identical laboratory FOG setups and validated our model. Exposed temperature profile ranges from $-40{ }^{\circ} \mathrm{C}$ up to $+60{ }^{\circ} \mathrm{C}$ under temperature rates between $\pm 0.2{ }^{\circ} \mathrm{C} / \mathrm{min}$.

In the literature, the quadrupole pattern is approached to be ideally symmetric through the coil. However, this symmetry is degraded due to practical necessities. In this letter, we built a model covering the properties of practical quadrupole pattern. Our model includes the fact that the length of the turn increases from inner to the outermost layer. Secondly, the first and last turns of each layer create axial asymmetry increasing the thermally induced error. Furthermore, fiber turns are wound in orthocyclic manner. Fiber coil is modeled as a transversely isotropic structure. We calculated the elastooptical interactions by homogenization/dehomogenization processes of representative volume element (RVE).

We built a Finite Element Method (FEM) model in two stages: the RVE structure and the whole fiber coil. Detailed fiber model defines the interactions inside RVE consisting fiber core, cladding, coating and adhesive. Homogenization and dehomogenization parameters of the RVE are obtained by simulations. Second model provides the temperature and strain distributions by using the homogenized fiber coil model along with the spool and environment. A temperature profile which is ranging from $-40{ }^{\circ} \mathrm{C}$ up to $+60{ }^{\circ} \mathrm{C}$ is applied to the model so that fiber coil is exposed to temperature fluctuations. Under this profile, temperature and stress field distributions are obtained for each fiber turn and each time instant. Local strain fields inside the fiber core are calculated by dehomogenization process using the global stress and temperature fields. Time derivatives of local temperature and strain fields are used to calculate the Shupe and elastooptic errors, respectively.

*E-mail: sogut@bilkent.edu.tr

Fiber Optic Sensors and Applications XIV, edited by Christopher S. Baldwin, Gary Pickrell,

Henry H. Du, Eric Udd, Jerry J. Benterou, Anbo Wang, Proc. of SPIE Vol. 10208, 1020806

(C) 2017 SPIE · CCC code: $0277-786 X / 17 / \$ 18 \cdot$ doi: 10.1117/12.2258030 


\section{THEORY}

Thermal fluctuations create nonreciprocal phase shift between counterpropagating waves in the fiber coil. Non-

reciprocal phase shift is defined by Shupe. ${ }^{1}$ Combining his equation with Sagnac relation, bias error $\Omega_{S}(t)$ for a FOG can be written as:

$$
\Omega_{S}(t)=\frac{n}{L D}\left(\frac{\partial n}{\partial T}+n \alpha\right) \int_{0}^{L} \dot{T}(z, t)(L-2 z) d z
$$

where $L$ is the length of the fiber, $D$ is the diameter of fiber coil, $n$ is the refractive index of fiber core, $\frac{\partial n}{\partial T}$ is the temperature coefficient of $n, \alpha$ is the thermal expansion coefficient of fiber core and $\dot{T}(z, t)$ is the temperature field time derivative and $z$ is the fiber portion where temperature fluctuates.

Ref. 3 extends the analysis for elastooptic interactions in fiber coil. It is shown that a change of the stress fields results bias error, $\Omega_{E} O(t)$ in FOG output. Relation can be written as follows:

$$
\Omega_{E O}(t)=\frac{n}{L D} \int_{0}^{L}\left[A \dot{\varepsilon}_{z}(z, t)-B \dot{\varepsilon}_{r}(z, t)\right](L-2 z) d z
$$

where $\dot{\varepsilon}_{z}$ and $\dot{\varepsilon}_{r}$ are the time derivatives of the axial and radial strain fields inside the fiber core, respectively, $A=n\left(1-\frac{1}{2} n^{2} p_{12}\right)$ and $B=\frac{1}{2} n^{3}\left(p_{11}+p_{12}\right)$, where $p_{11}$ and $p_{12}$ are the photoelastic coefficients of the fiber glass.

These two main bias error mechanisms are additive. So the total bias error is

$$
\Omega_{\text {Total }}(t)=\Omega_{S}(t)+\Omega_{E O}(t)
$$

\section{HOMOGENIZATION AND DEHOMOGENIZATION OF FIBER COIL}

Fiber coil consists of fiber turns, potting material between the turns and a spool. Fiber itself consists of core, cladding and coating. Excluding the spool, RVE for fiber coil structure is obtained for homogenization/dehomogenization processes. ${ }^{4,5}$ After obtaining the homogenized fiber coil model, FEM environment is built with spool. Global temperature and strain fields are obtained by FEM simulations. Lastly, dehomogenization procedure is carried. In this stage, global fields are mapped to local fields which are used for the calculation of bias error.

Fiber coil is an orthotropic and inhomogeneous structure. A homogenization process is performed by defining RVE (Fig. 1). We used a fiber which is reduced cladding and double-coated. The diameter of cladding is 80 $\mu \mathrm{m}$. That of the first and second coating layers are $130 \mu \mathrm{m}$ and $170 \mu \mathrm{m}$, respectively. We built three different boundary conditions in order to calculate homogenization parameters for our fiber model. The results are given in Tab. 1.

Table 1: Coil parameters obtained by homogenization.

\begin{tabular}{lll}
\hline Parameters & Direction & Value \\
\hline \hline Elastic moduli & $E_{z}(\mathrm{GPa})$ & 14.5 \\
& $E_{r}(\mathrm{MPa})$ & 95.2 \\
& $G_{z r}(\mathrm{MPa})$ & 24.1 \\
\hline Poisson's ratio & $\nu_{z r}$ & 0.392 \\
& $\nu_{r}$ & 0.979 \\
& $\nu_{r z}$ & 0.003 \\
\hline Thermal expansion coefficient & $\alpha_{z}\left(10^{-6} / \mathrm{K}\right)$ & 3.36 \\
& $\alpha_{r}\left(10^{-6} / \mathrm{K}\right)$ & 193 \\
\hline Thermal conductivity & $\kappa_{z}(\mathrm{~W} / \mathrm{mK})$ & 0.51 \\
& $\kappa_{r}(\mathrm{~W} / \mathrm{mK})$ & 0.34 \\
\hline
\end{tabular}




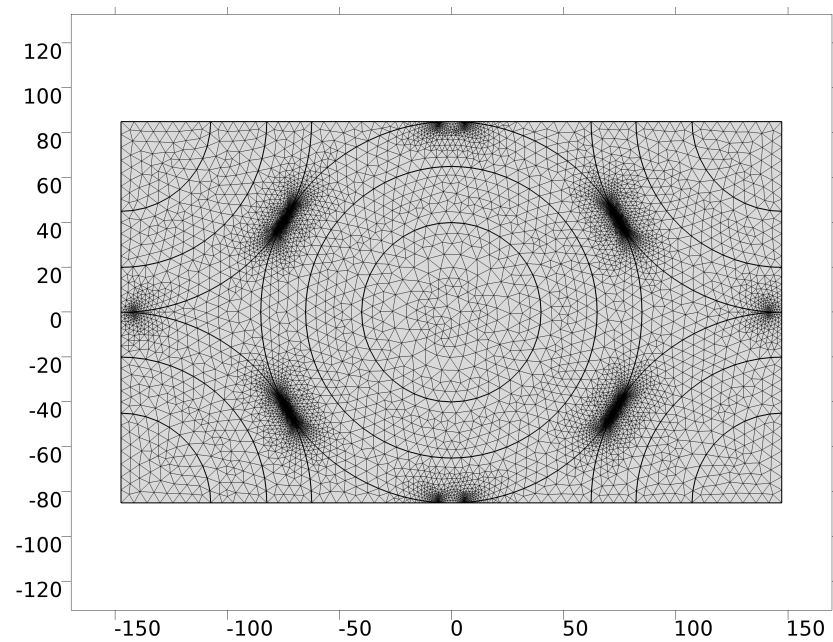

Figure 1: Simulation of RVE with high resolution mesh. Fibers located orthocyclic manner with adhesive in between. All dimensions are in $\mu m$.

The homogenization parameters are used for fiber coil simulations. However, FEM simulation outputs macroscopic temperature and strain fields. Strain fields inside the core are calculated by dehomogenization process. Dehomogenization of the global fields is a transfer function, as follows:

$$
\left(\begin{array}{l}
\bar{\varepsilon}_{r, F} \\
\bar{\varepsilon}_{z, F}
\end{array}\right)=M\left(\begin{array}{c}
\bar{\varepsilon}_{x x} \\
\bar{\varepsilon}_{y y} \\
\bar{\varepsilon}_{z z} \\
\Delta T
\end{array}\right)
$$

where $\bar{\varepsilon}_{r, F}$ and $\bar{\varepsilon}_{z, F}$ are strain fields inside the core in radial and longitudinal directions, respectively; $\bar{\varepsilon}_{x x}$, $\bar{\varepsilon}_{y y}$ and $\bar{\varepsilon}_{z z}$ are the global strain fields obtained by FEM simulation in there dimensions and $\Delta T$ is the local temperature difference. $M$ is the transformation matrix,

$$
M=\left[\begin{array}{cccc}
M_{r x} & M_{r y} & M_{r z} & M_{r T} \\
0 & 0 & 1 & 0
\end{array}\right]
$$

Transformation matrix elements are obtained along with homogenization process. Calculated matrix elements are given in Tab. 2. Finally, microscopic strain fields are obtained.

Table 2: Dehomogenization parameters.

\begin{tabular}{l|l}
\hline$M_{r x}$ & $2.74 \cdot 10^{-6}$ \\
\hline$M_{r y}$ & $2.70 \cdot 10^{-6}$ \\
\hline$M_{r z}$ & -0.17 \\
\hline$M_{r T}$ & $9.98 \cdot 10^{-6} 1 / K$ \\
\hline
\end{tabular}

\section{SIMULATION}

A fiber coil is designed with the parameters given in Tab. 3. FEM simulation is performed in order to obtain the temperature and strain fields. FEM model consists of the homogenized fiber coil wounded on an aluminum spool. Heat source provides a temperature profile ranging from $-40{ }^{\circ} \mathrm{C}$ to $60^{\circ} \mathrm{C}$. This profile reveals all temperature and temperature time derivative dependent errors in the interval. Simulation calculates the heat flow according to the material properties of the spool and the fiber coil. 
Table 3: Fiber coil design.

\begin{tabular}{l|l}
\hline Fiber length & $1101.57 \mathrm{~m}$ \\
\hline Number of winding layers & 36 \\
\hline Number of loops per layer & 106 \\
\hline Inner diameter of the coil & $87.00 \mathrm{~mm}$ \\
\hline Outer diameter of the coil & $97.65 \mathrm{~mm}$ \\
\hline Coil height & $18.02 \mathrm{~mm}$ \\
\hline
\end{tabular}

Microscopic strain and temperature fields are used as inputs of Eqs. (1) and (2), along with distance of each fiber turn from the fiber end point. Distance of each turn is calculated using the practical quadrupole cross section view (Fig. 2).

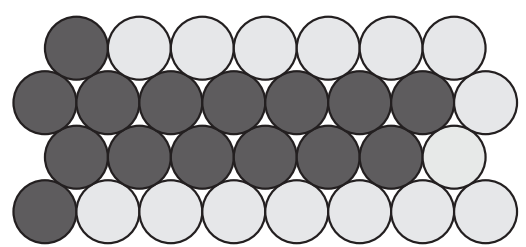

Figure 2: Practical quadrupole pattern.

Practical quadrupole winding has some major differences from ideal quadrupole. Firstly, the length and diameter of turns differ in each layer of the fiber coil, which creates additional radial asymmetry. Secondly, first turn of each layer is wound either clockwise (CW) or counter-clockwise (CCW) and last turn vice versa. Therefore, fiber coil shows a figure with each side is either CW or CCW wound. This is a practical solution for passing the fiber from a layer to the next one. This second difference creates an axial asymmetry. We note that axial asymmetry is not negligible especially under thermal stress. Lastly, fiber turns are located in an orthocyclic manner.

Temperature distribution in time for each fiber turn is given in Fig. 3. Bias error due to Shupe effect is calculated using this distribution. Microscopic strain fields obtained after dehomogenization are given in Fig. 4. The time derivatives of the fields are used in the calculation of the elastooptical bias error. Fig. 5 shows Shupe and elastooptic bias errors. We note that elastooptical error is greater than the pure Shupe error.

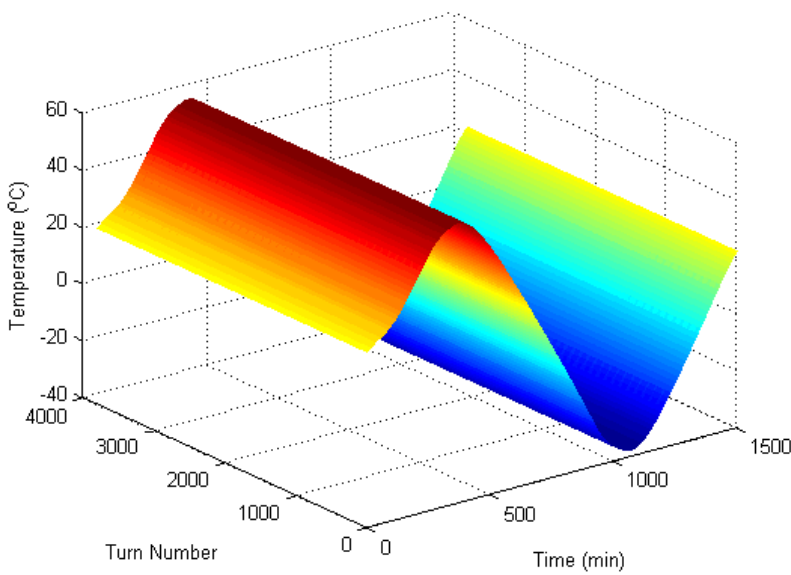

Figure 3: Temperature distribution along fiber coil. 


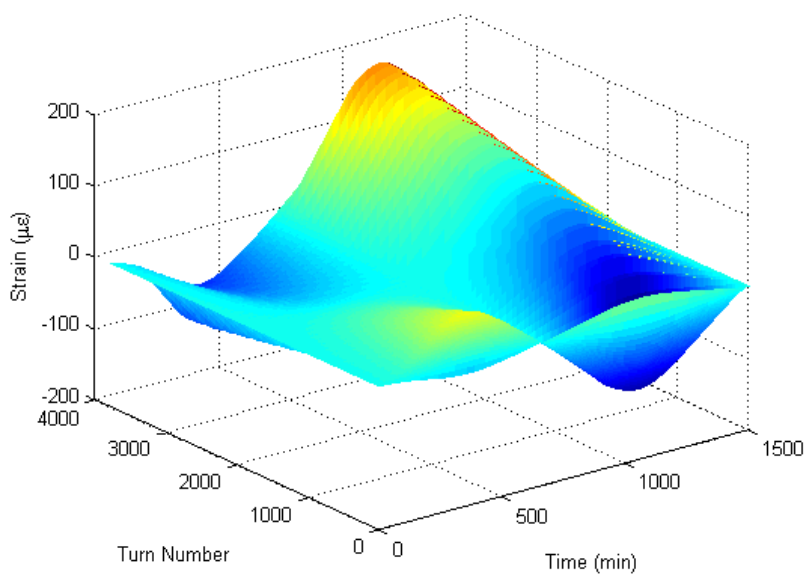

(a) Radial strain.

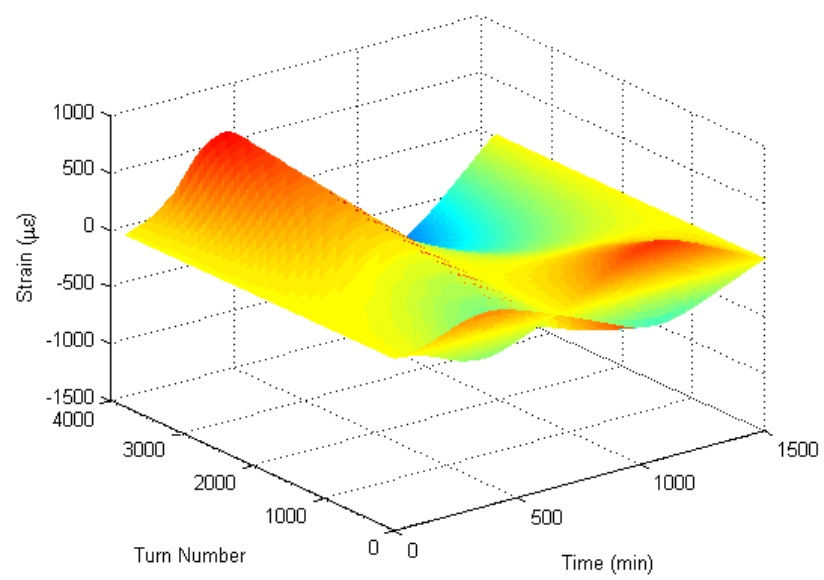

(b) Axial strain.

Figure 4: Strain distributions along fiber coil.

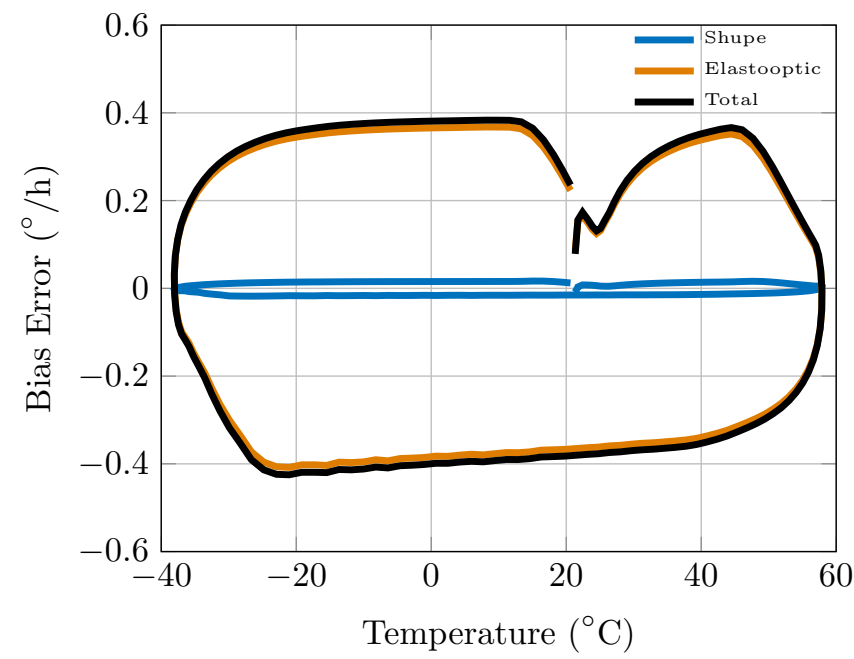

Figure 5: Bias errors due to Shupe and elastooptic effects.

\section{EXPERIMENT}

Experiments are carried with three fiber coils having same design parameters as in simulations. Temperature sensors are mounted on each coil spool to monitor the coil temperature. Temperature profile ranging from $-40{ }^{\circ} \mathrm{C}$ to $+60{ }^{\circ} \mathrm{C}$ is applied to fiber coils while temperature and rate measurement data are collected. Collected rate data is processed for eliminating the earth rotation and gyro noise. Experimental results of three coils and simulation result are plotted in Fig. 6. The ratio of bias error to temperature derivative is defined as thermal sensitivity coefficient. Calculated coefficients are given in Tab. 4.

Table 4: Thermal sensitivity coefficients.

\begin{tabular}{|c|c|}
\hline Coil No & $\begin{array}{l}\text { Thermal Sensitivity Coef. } \\
\left(\frac{{ }^{\circ} / h}{{ }^{\circ} \mathrm{C} / \mathrm{min}}\right)\end{array}$ \\
\hline Coil-1 & 3.01 \\
\hline Coil-2 & 1.39 \\
\hline Coil-3 & 1.98 \\
\hline Simulation & 2.71 \\
\hline
\end{tabular}




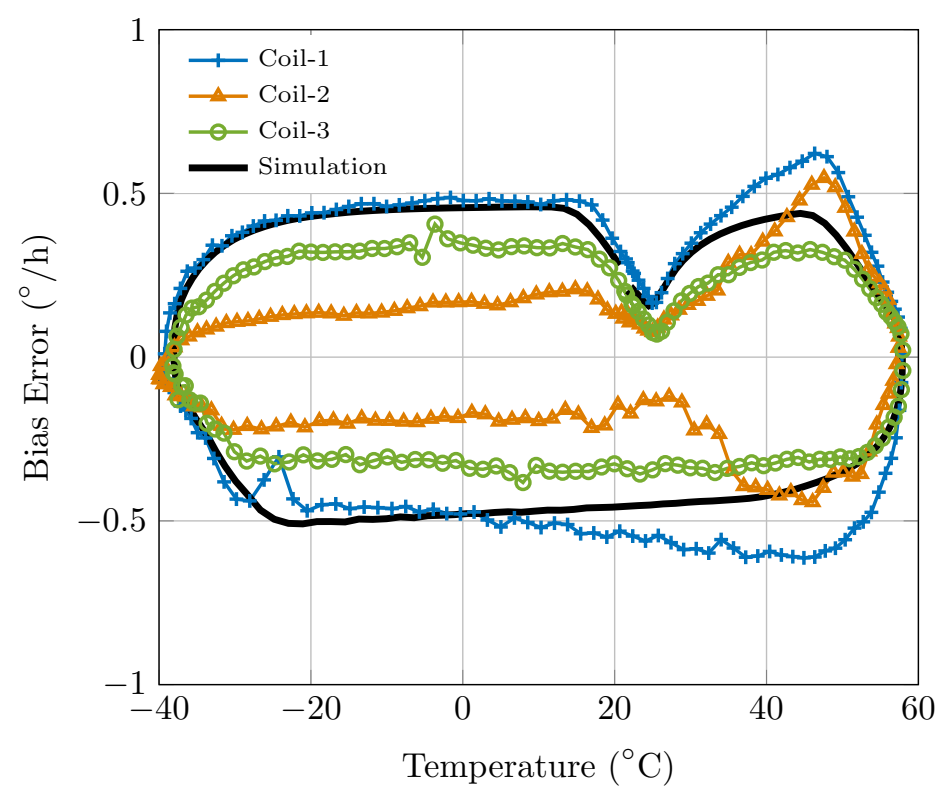

Figure 6: Simulated and experimental bias error curves.

Bias error characteristics for three fiber coils are consistent with theoretical model. Difference between the sensitivity coefficients of the coils could be a result of fiber tail length asymmetry during the production of coils. Also a change of the amount of the adhesive during the production could be another reason. It is also seen that sensitivity coefficients of Coil- 1 and Coil- 2 change for different temperatures. This result can be explained by temperature dependent material properties.

\section{CONCLUSION}

In this letter, we built an advanced fiber coil model covering the Shupe effect, elastooptical interactions, orthotropic coil model and practical quadrupole winding pattern. We simulated a temperature fluctuation scenario on model and calculated the bias errors. We report experiments of three identically designed fiber coils and comparison of the results with simulation calculations. Experimental and simulation results are consistent. As a future work, simulations and experiments with different coil geometries, adhesives and spool materials are planned to be carried out.

\section{REFERENCES}

[1] Shupe, D. M., "Thermally induced nonreciprocity in the fiber-optic interferometer," Appl. Opt. 19(5), 654655 (1980).

[2] Frigo, N. J., "Compensation of linear sources of non-reciprocity in sagnac interferometers," Proc. SPIE 0412, 268-271 (1983).

[3] Mohr, F. and Schadt, F., "Bias error in fiber optic gyroscopes due to elasto-optic interactions in the sensor fiber," Proc. SPIE 5502, 410-413 (2004).

[4] Schadt, F., Physikalische Modellierung und Analyse thermisch und mechanisch bedingter Messabweichungen in faseroptischen Rotationssensoren, Ph.D. dissertation, University Magdeburg (2002).

[5] Minakuchi, S. et al., "Thermal strain in lightweight composite fiber-optic gyroscope for space application," J. Lightwave Technol. 33(12), 2658-2662 (2015). 